\title{
Updates from the ANITA Experiment
}

\author{
Cosmin Deaconu for the ANITA collaboration ${ }^{1, \star}$ \\ ${ }^{1}$ Kavli Institute for Cosmological Physics, University of Chicago \\ 5640 South Ellis Ave, Chicago, Illinois, United States of America
}

\begin{abstract}
.
The ANtarctic Impulsive Transient Antenna (ANITA) collaboration deploys balloonborne interferometric antenna payloads that fly at $37 \mathrm{~km}$ above Antarctica. The primary goal is detection of Askaryan emission from cosmogenic neutrinos interacting in the Antarctic ice. In addition, ANITA has proven sensitive to radio signals from extended air showers. Here, we provide a review of the results of previous missions, with a special focus on recent results, and a preview of our upcoming mission.
\end{abstract}

\section{Introduction}

Cosmogenic neutrinos [1] from the interaction of the Ultra-High-Energy Cosmic Ray (UHECR) protons with the cosmic microwave background[2] are expected to exist at EeV energies but have yet to be detected. The low flux and small interaction cross-sections pose formidable challenges, but successful detection would provide new information both about astrophysical sources of UHECR's as well as the highest energy measurement of neutrino-nucleon interactions.

Economic instrumentation of large target material volumes may be accomplished by exploiting the Askaryan effect[3]. The interaction of an extremely-high-energy neutrino in a dielectric, such as ice, produces a moving charge excess resulting in electromagnetic emission that becomes coherent at wavelengths larger than the transverse size of the avalanche $(<\sim \mathrm{GHz})$. This emission has been confirmed in laboratory beamline tests[4] and is not greatly attenuated in media such as glacial ice, allowing large acceptance volumes with few antennas.

The ANtarctic Impulse Transient Antenna (ANITA) long-duration interferometric balloon payload scans the Antarctic continent for impulsive radio-frequency emission produced by neutrino interactions in the ice. Antarctica is the ideal location for the payload due to its preponderance of ice, comparatively-few anthropogenic sources of background, and high-altitude air currents suitable for long-duration ballooning. At the $37 \mathrm{~km}$ float altitude, roughly one million cubic kilometers of ice are visible at a time, but the large distance from the interactions implies a high threshold $(\sim \mathrm{EeV})$.

As a broadband impulse radio detector, ANITA is also sensitive to radio-emission from extended air showers (EAS), which is primarily synchrotron emission from charge-separation by the Earth's magnetic field[5]. This allows ANITA to directly measure UHECR's of sufficient energy and also provides sensitivity to another channel for detection of cosmogenic neutrinos - a $v_{\tau}$ may interact in the Earth producing a $\tau$ that can escape and hadronically decay in the atmosphere, creating an upward-going EAS.

^e-mail: cozzyd@kicp.uchicago.edu 


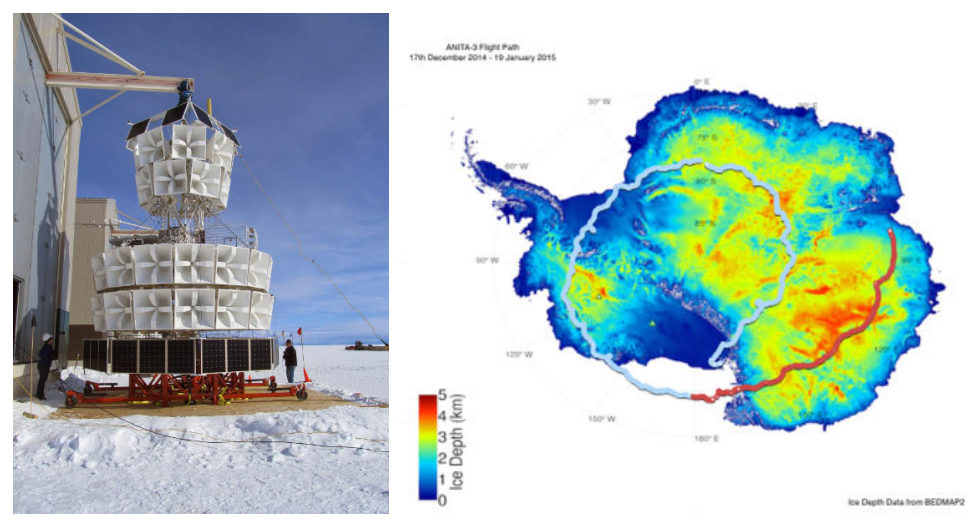

Figure 1. The ANITA-3 payload (left) and flight path (right). The vertical axis denotes the ice depth and the change in color of the flight path occurs at the second revolution around McMurdo

\section{Instrument}

As a balloon payload in a remote environment, ANITA must be capable of running with minimal interaction and comply with severe weight and power consumption limitations. ANITA's sensitive band is roughly 200-1300 MHz. ANITA payloads consist of 32-48 dual-polarization high-gain antennas, tilted slightly downward towards the horizon to maximize acceptance. The signal from antennas is amplified and then split into digitization and trigger circuits.

LAB4 switched-capacitor arrays [6] are used for digitization due to their their high sampling rate $(2.6 \mathrm{GSa} / \mathrm{s})$ and low power requirements. However, this comes at the cost of slow readout and complicated calibration for the analog buffers. Multiple chips are used for each channel to minimize the effect of the long-readout time on deadtime.

Tunnel diode square-law detectors form the basis of the trigger. Onboard field-programmable gate arrays combine individual channel level crossings into global triggers that result in digitization. The exact trigger strategy has varied from flight to flight. Thresholds are dynamically set so that the instrument is taking as much data as possible without significant deadtime. A maximum sustained global trigger rate of $\sim 50 \mathrm{~Hz}$ is achievable.

So far there have been three flights of the ANITA payload. The most recent flight, ANITA-3 (Fig. 1), flew in the 2014-2015 season for 22 days with 48 antennas. The flight had to be terminated relatively quickly due to wind patterns that threatened to send the payload off the continent. Unlike previous flights which had split the signal into multiple frequency bands and required signal above threshold in each band, a full-band trigger in both vertical and horizontal polarizations was used. This was expected to improve sensitivity at low signal-to-noise ratio, but also proved much more susceptible to triggering on man-made continuous-wave $(\mathrm{CW})$ emissions. These unwanted triggers resulted in high dead-time on the side of the payload pointing North. The analysis from ANITA-3 is still ongoing as of writing.

The fourth flight of ANITA is expected this Austral winter. Upgrades include dynamic notch filtering to combat $\mathrm{CW}$ from satellites, a higher-altitude flight, improved amplifiers, and new trigger logic. Phase shifters will be used to convert linear to circular polarization and coincidence between LCP and RCP will be required. As a result, ANITA will have equal acceptance to linearly polarized radiation in any polarization plane and be less likely to trigger on the mainly circularly polarized signals from satellites. As in ANITA-3, a GPU-based online event prioritizer will be used to select 


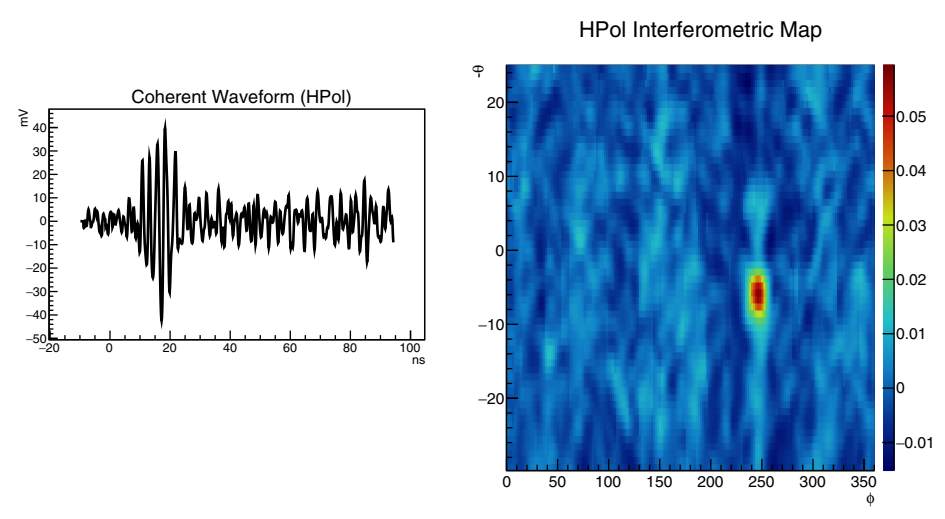

Figure 2. An example coherently-summed waveform (left) and interferometric map (right) from a horizontally-polarized calibration pulser during the ANITA-3 flight.

which events will be telemetered to ground in case the full dataset cannot be recovered from the payload.

\section{Calibration and Analysis}

In order to help characterize the instrument, high-voltage pulsers transmit impulsive signals to the instrument from various sites on the Antarctic continent. This allows calibration of the antenna positions as they are in flight and allows estimation of trigger efficiency and pointing resolution as a function of signal-to-noise ratio. For ANITA-3, the Hi-Cal trailing balloon provided a balloon-borne high-voltage impulse calibration signal for a portion of the flight. Both the direct and ice-reflected signals from Hi-Cal can be measured. A similar balloon-based calibration is planned to be repeated for ANITA-4.

ANITA analyses have generally followed a similar procedure. First, the waveforms from each event are filtered to remove anthropogenic CW contamination from satellites and bases. Then the likely primary direction of the signal by using an interferometric method [7] considering crosscorrelations between antennas. Fig. 2 shows an example interferometric map from a calibration pulser event. Cuts are then applied to remove remaining CW, events likely to be thermal noise (where the signals between antennas will be uncorrelated) and other pathological events, leaving only candidate physics events and anthropogenic activity. Spatial clustering is used to group remaining events into known-bases or apparent hot-spots of activity. Any spatially isolated events are considered physics candidates. The isolated anthropogenic background is estimated from the number of small-event clusters.

\section{Science Results}

A cosmogenic neutrino search seeks primarily vertically-polarized isolated impulsive emission that may be a result of Askaryan emission. This preferred plane of polarization is expected from geometric acceptance considerations and transmission at the ice-air interface. ANITA-2 has the best current limits on cosmogenic neutrinos in the $10^{21} \mathrm{eV}$ range [8].

Due to nearly vertical magnetic field in the Antarctic region, synchrotron emission from EAS is expected to be primarily horizontally polarized. ANITA-1 measured 16 such events[5]. Because ANITA-2 only triggered on vertical polarization, it was much less sensitive to EAS and only detected 

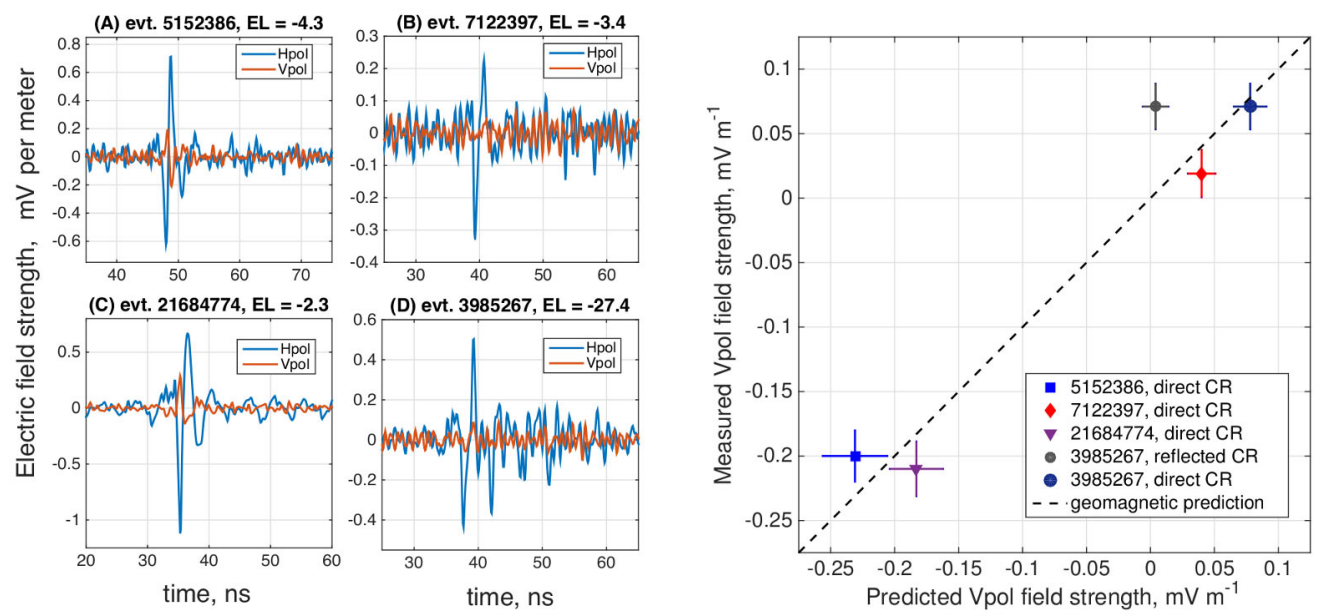

Figure 3. (Left) Coherently-summed waveforms from the three apparent Earth-skimming extended air showers from ANITA-1 and 2, as well as the non-inverted EAS candidate coming from the Earth. The instrument response has been deconvolved. (Right) The predicted versus measured vertical-polarization component of the events, including two assumptions from the apparent upward event.

three. Due to ANITA's high altitude, most EAS development occurs below the payload and the emission measured is reflected off the ice, which results in an apparent incoming direction from the ground and signal polarity inversion. However, ANITA has also detected nearly horizontal showers where the shower misses the Earth entirely; in these cases the signal comes from above the horizon there is no signal inversion.

The geomagnetic origin of these events can be further tested by comparing the measured polarization plane with the expected plane defined by the Earth's local magnetic field, the cosmic ray direction, and, in the case of reflected events, the appropriate Fresnel coefficients. Agreement between prediction and measurement is confirmed for the events from ANITA-1 and 2.

An additional isolated horizontally-polarized event from ANITA-1 [9] has a non-inverted signal shape characteristic of the Earth-skimming EAS's, but appears to come from well below the horizon (Fig. 3). In addition, the geomagnetic polarization plane analysis is also more consistent with no reflection off the ice. This event is challenging to interpret. While it is impossible to rule out anthropogenic origin, only 1.6 events were expected to pass clustering cuts. Only a few percent of all anthropogenics have a shape with as high a cross-correlation coefficient with the average EAS waveform shapes as this event. Moreover, only half would have the correct polarity and most anthropogenics are not expected to have polarization angle so close to the geomagnetic prediction. None of these factors, other than clustering, were used in the event selection.

A potential physics explanation is an upward-going EAS from the hadronic decay of a $\tau$ produced by the interaction of a EeV-scale $v_{\tau}$ in the crust or ice. However, the elevation angle is such that the chord through the Earth would be roughly $5500 \mathrm{~km}$. The projected Standard-Model cross-section for neutrinos at these energies through the Earth predicts an attenuation factor on the order of $10^{-4}$ for this much material[10]. If this event truly is a $\tau$-induced air shower, it would be in significant tension with the Standard Model. The extremely-high-energy neutrino cross-section reduction on the order of 3-5 would be required to plausibly accommodate such an event. If this really were the case, the acceptance 
calculations that previous neutrino limits are based on would need to be updated. ANITA-4 is moving to a polarization-plane-blind trigger partially for this reason.

\section{Conclusion}

The ANITA program has had three successful flights. The analysis of the third flight is still ongoing. Due to triggering on horizontally-polarized events, it is expected to measure additional EAS events, perhaps shedding additional light on the unexpected event from ANITA-1. ANITA-4 is to fly soon, which will address many of the shortcomings of the previous flight. ANITA acknowledges generous support from NASA, the Columbia Scientific Balloon Facility, the National Science Foundation, and the Department of Energy, High Energy Physics Division.

\section{References}

[1] V.S. Berezinsky, G.T. Zatsepin, Phys. Lett. B28, 423 (1969)

[2] K. Greisen, Phys. Rev. Lett. 16, 748 (1966)

[3] G.A. Askar'yan, Sov. Phys. JETP 14, 441 (1962), [Zh. Eksp. Teor. Fiz.41,616(1961)]

[4] P.W. Gorham et al. (ANITA), Phys. Rev. Lett. 99, 171101 (2007), hep-ex/0611008

[5] S. Hoover et al. (ANITA), Phys. Rev. Lett. 105, 151101 (2010), 1005.0035

[6] G.S. Varner, L.L. Ruckman, P.W. Gorham, J.W. Nam, R.J. Nichol, J. Cao, M. Wilcox, Nucl. Instrum. Meth. A583, 447 (2007), physics/0509023

[7] A. Romero-Wolf et al., Astropart. Phys. 60, 72 (2015)

[8] P.W. Gorham et al. (ANITA), Phys. Rev. D82, 022004 (2010), [Erratum: Phys. Rev.D85,049901(2012)], 1011.5004

[9] P.W. Gorham et al., Phys. Rev. Lett. 117, 071101 (2016), 1603.05218

[10] E. Bugaev, T. Montaruli, Y. Shlepin, I.A. Sokalski, Astropart. Phys. 21, 491 (2004), hep-ph/0312295 